\title{
EVALUACIÓN AMBIENTAL DEL PLAN DE DESARROLlO DE LA CIUDAD DE ARMENIA EN EL PERIODO 2008-2011
}

\section{ENVIRONMENTAL EVALUATION OF THE 2008-2011 DEVELOPMENT PLAN OF ARMENIA}

Luz Elena García García*

José Fabián Ríos Obando**

\begin{abstract}
RESUMEN
$E^{1}$ presente artículo se deriva de los resultados de una investigación descriptiva cuyo objetivo es evaluar el Plan de Desarrollo Municipal de Armenia desde la perspectiva ambiental, en el Periodo 2008-2011. La metodología de Gestión Integral de Programas Sociales Orientadas a Resultados, propende por reconocer si las acciones implementadas a partir de la aplicación del instrumento de política ha ocasionado un cambio importante en las condiciones de vida de la población. La técnica entonces consistió en implementar indicadores en dos momentos: la línea base (año 2007) y el periodo de tiempo analizado (2008-2011), en aras de establecer mediante la comparación entre ambos escenarios, un posible cambio en las condiciones de vida de la población. Los resultados y conclusiones del proyecto posibilitaron identificar hechos que brindaron condiciones ambientales en Armenia.
\end{abstract}

Palabras clave: ambiental, condiciones de vida, dimensión, sostenibilidad, planes de desarrollo.

* Luz Elena García García, Economista, Universidad de Manizales; Magíster en Pedagogías Activas y Desarrollo Humano, Alianza Universidad de Manizales-CINDE; Doctora en Conocimiento y Cultura en América Latina, Instituto Pensamiento y Cultura en América Latina - IPECAL (México). Docente-Investigadora Centro Grupo de Investigaciones en Desarrollo y Medio Ambiente (CIMAD), Universidad de Manizales, Manizales, Caldas, Colombia. Correo electrónico: luzeg@umanizales.edu.co.

** José Fabián Ríos Obando, Administrador de Empresas. Docente-investigador Corporación Universitaria Centro Superior Unicuces. Articulo derivado de la investigación como opción de grado titulada "Determinación del impacto económico y ambiental del plan de desarrollo de la ciudad de Armenia en el periodo 2008-2011", el cual se encuentra en el repositorio RIDUM de la Universidad de Manizales. Correo electrónico: invrios@unicuces.edu.co. 


\section{ABSTRACT}

This article is derived from the results of a descriptive research aimed at assessing the $\mathrm{Mu}-$ nicipal Development Plan of Armenia from an environmental perspective, in the period 2008-2011.

The methodology for Comprehensive Management of Social Programs Results Oriented, tends to recognize whether the actions implemented from the implementation of the policy instrument has caused a major change in the living conditions of the population. The technique was then to implement indicators into two moments-based (2007) line and the time period analyzed (2008-2011) -, in order to establish by comparing two scenarios, a possible change in living conditions of the population.

The findings and conclusions of the project made it possible to identify events that provided environmental conditions in Armenia.

Keywords: development plans, dimension, environment, living conditions, sustainability

\section{INTRODUCCIÓN}

Las decisiones políticas tomadas por el Estado son orientadas en su mayoría bajo criterios netamente económicos, bajo el supuesto de que tras obtener beneficios macroeconómicos, los mismos redundaran en beneficios para la sociedad en conjunto. Se observa entonces un desconocimiento de la inter dependencia de los factores sociales y ambientales como participes de las dimensiones que garantizan la sostenibilidad.

Al analizar la compleja realidad en la que estamos sumidos se observa que el Estado aun cuando tenga como fin constitucional garantizar calidad de vida para los ciudadanos, tiene la tendencia a fijar posiciones concibiendo al aspecto económico como eje central, en la cual los demás factores han de ajustarse en torno a él para que mediante los instrumentos de mercado (propios de la economía contemporánea) repercutan en condiciones favorables para el bien público. Es por tal situación que diversos autores, entre los que se destaca José Manuel Naredo, han considerado que el grado de correlación entre las decisiones económicas ajustada a criterios sociales y ecológicos es mínima, toda vez, que dentro de la formulación de políticas públicas, no se estudia a fondo las implicaciones sobre la biodiversidad o las mismas no responden a condiciones que contribuyan a resarcir y compensar al ecosistema o comunidades afectadas.

Igualmente es preocupante observar en los procesos de formulación de políticas públicas, la escasa participación de la ciudadanía, la cual ha sido tradicionalmente marginada de ejercer un derecho constitucional. Es necesario entonces garantizar a todo nivel un proceso de inclusión que propicie la activa participación de la comunidad, principalmente aquellas con mayor grado de vulnerabilidad, para que, a partir de sus percepciones coadyuven a estructurar proyectos con mayor carga social, los cuales respondan a sus necesidades, siendo entonces prioritario promover proyectos que particularicen a cada uno de los sectores o comunidades, bajo el entendido de que al generalizar las necesidades 
de una sociedad se desconoce que cada comunidad en su singularidad tienen necesidades que divergen de otros grupos o nichos urbanos y que por ende merecen un análisis específico para apuntar a que partir de procesos incluyentes se dote a la población de las libertades y capacidades que ellos consideran apremiantes.

El modelo económico imperante, al cual se responsabiliza por la acelerada destrucción de los recursos naturales y los álgidos problemas de índole social hace necesario configurar un nuevo orden que responda a las problemáticas en comento, de forma tal que sean los conceptos proferidos por la ecología y economía ambiental los que constituyan el eje transversal que permita incluir a la sociedad en la toma de decisiones para dar paso a los mecanismos y métodos de la ciencia económica que permitan alcanzar niveles de desarrollo deseados bajo los principios de la sostenibilidad.

Los cuestionamientos hacia la economía clásica y su esterilidad hacia el reconocimiento de las condiciones ambientales, dieron lugar a una estructura de pensamiento subyacente a un ethos particular a la segunda mitad del siglo XX, el cual motivado por diversas concepciones; como el desarrollo a escala humana, donde Neff (1994) vuelca la atención, hacia el ser humano como eje dinamizador de la economía, de esta manera su postulado se centraba en considerar que el desarrollo debe girar en torno a las personas.

Del mismo modo, coexisten otras cosmovisiones de sociedad, como las aportadas por Sen (2000), el cual sentó las bases de una nueva economía basada en la satisfacción de las necesidades, la cual ha de dotar al individuo de una serie de potencialidades y capacidades que le restituyan la libertad y capacidad de toma de decisiones, como única posibilidad de eliminar la pobreza.

Casi que de forma paralela a las consideraciones sociales, se gestó un discurso, nutrido por autores como Lovelock (1972), para el cual la tierra como ecosistema, se auto-regula y permite que las condiciones de vida se mantengan. De acuerdo con la denominada teoría Gaia, para garantizar un sistema en equilibrio, se debe conservar el mayor número y cantidad posible de especies; ante tal presupuesto, se debe garantizar la protección de la biodiversidad y del ecosistema, como mecanismo para preservar un sistema en homeostasis.

Durante los últimos decenios, desde la academia y otros sectores se ha demostrado sistemáticamente la urgencia de un cambio en la estructura economía y política, la cual ha causado una escisión entre hombre-naturaleza y que se ve reflejada mediante diversos tipos de impactos, como el incremento exponencial de residuos que logra saturar los rellenos sanitarios, contaminación del nivel freático y otros problemas asociados al uso intensivo de recursos naturales.

La perspectiva ambiental del desarrollo emerge así como una nueva visión del proceso civilizatorio de la humanidad. La crisis ambiental vino a cuestionar las bases conceptuales que han impulsado y legitimado el crecimiento económico, negando a la naturaleza. La sustentabilidad ecológica aparece como un criterio normativo para la reconstrucción del orden económico, como una condición para la sobrevivencia humana y para el logro de un desarrollo durable, problematizando los valores sociales y las bases mismas de la producción (Leff, 2011, p.10) 
De acuerdo a Redclift (1996) "existe la necesidad de reconocer el concepto de desarrollo sostenible como alternativa a la visión establecida, y no como modificación de la misma".

Dichas situaciones se replican en los diferentes escenarios, para el caso en particular, analizando el contexto nacional y local se puede afirmar que la ciudad de Armenia no es ajena a dicha realidad, en la que se observan álgidos problemas de diversa índole, como el bajo nivel de escolaridad de la población, altas tasas de desempleo, pobreza extrema, entre otros y que son el reflejo de la marginación de la población dentro de la estructura económica, política y social.

Se observa entonces la incapacidad del Ente Territorial para abordar de forma expedita y coherente las problemáticas que subsisten en torno a las dimensiones de la sostenibilidad y poder así lograr consolidar un desarrollo que responda entre otros criterios a atender las necesidades de la población más vulnerable.

De acuerdo con Naredo (2009) no existe algún tipo de correlación entre las decisiones económicas en relación a criterios sociales y ecológicos, por cuanto en las discusiones e implementación de la agenda pública, poco se analiza los impactos antropogenicos y externalidades causadas al ecosistema, mientras que en otros casos, las medidas de mitigación no responden a condiciones que contribuyan resarcir y compensar al ecosistema o comunidades afectadas

Urge entonces una visión de sostenibilidad que garantice la protección de los recursos naturales, como una apuesta por lograr una equidad intra e inter generacional, principalmente hacia la sociedad vulnerable.

Ante tal situación es necesario realizar investigaciones al Plan de Desarrollo a la ciudad de Armenia, de forma que a partir de los resultados observados se logren construir de forma colectiva propuestas que apunten a solucionar los aspectos críticos que sean revelados a partir del ejercicio puesto a consideración.

Los planes de desarrollo como instrumento de planeación deben encargarse de trazar una visión de largo plazo de la ciudad como única posibilidad de dar respuesta a problemas estructurales mencionados previamente. Para tal fin es necesario entonces apostar por procesos de largo aliento que den cuenta de la compleja realidad en la que nos encontramos y de los grandes retos que esto genera. Sin embargo, la experiencia nos demuestra que el concepto de política pública se ha perdido por completo, entonces nos enfrentamos a políticas de gobierno, las cuales cada periodo legislativo sufren cambios ostensibles y en muchos casos parten de cero, sin tomar como referencia los programas y proyectos que se encontraban en marcha, rechazando toda posibilidad de continuar con ciertos programas con nobles intenciones lo que termina por socavar toda posibilidad de dar una respuesta oportuna a las problemáticas abordadas.

La intención de un Plan de Desarrollo es lograr consolidar un escenario en el que los resultados alcanzados perduren en el tiempo y que los mismos logren permear a la sociedad en general, en aras de lograr una correcta distribución de cargas y beneficios. 
Ahora bien, en lo que concierne a las evaluaciones sobre planes de desarrollo resulta imprescindible hacer relación al Informe del Departamento Nacional de Planeación (DNP), 2011 "Evaluación del Desempeño Integral de los Municipios", en el que se observa como el DNP viene desarrollando una metodología para la medición del desempeño de los municipios, dicho informe revela información sobre los resultados alcanzados conforme las metas establecidas en los planes de desarrollo(eficacia), la eficiencia(entendida como la relación optima entre productos alcanzados e insumos utilizados), cumplimiento de requisitos legales( marco normativo que regula los recursos del Sistema General de Participaciones) y por último la gestión administrativa y fiscal (la cual mide la capacidad administrativa y financiera para lograr las metas propuestas).

Luego de realizar un rastreo investigativo se observa tanto en el ámbito nacional como internacional un sinfín de evaluaciones realizadas a los planes de desarrollo, cuyas intenciones se han centrado en develar los resultados de la implementación de los planes de desarrollo a la luz de las metas y objetivos propuestos, así mismo se observan análisis detallados sobre la ejecuciones presupuestales con base a los planes de inversiones propuestos.

Se observó entonces que las evaluaciones realizadas a los planes de desarrollo consisten en evaluaciones a la gestión realizada durante el periodo legislativo dejando entrever que dichas investigaciones han desconocido la trascendencia de identificar los efectos de los programas y proyectos llevados a cabo con ocasión a los planes de desarrollo.

De conformidad con lo anterior toma relevancia la necesidad de realizar procesos investigativos que pretendan establecer mediante un análisis comparativo entre la situación base (inicial) y la situación final la causalidad entre las acciones emprendidas y los efectos observados.

En el marco del contexto nacional y local, se puede afirmar que en la ciudad de Armenia se observan álgidos problemas en el ecosistema y que son el síntoma de la marginación de la población dentro de la estructura económica, política y social.

El municipio de Armenia, capital del Departamento del Quindío, es una ciudad intermedia, ubicada en el denominado triángulo de oro, toda vez que se encuentra delimitada por un triángulo, cuyos vértices son las tres principales ciudades del país: Bogotá( Capital de Colombia), Medellín y Cali.

Las ciudades que hacen parte de dicho grupo son además de Armenia, otras ciudades capitales, donde se destacan: Manizales, Pereira e Ibagué

Se debe destacar que tales municipios logran agrupar alrededor del 56\% de la población total y el $76 \%$ del producto interno bruto (PIB).

Las coordenadas de Armenia son $45170^{\circ}$ de latitud norte, $756830^{\circ}$ oeste, ubicada aproximadamente a 290 kilómetros de Bogotá. De acuerdo con las proyecciones del Departamento Administrativo Nacional de Estadística (2010), la población al 2012 es de 292052 habitantes, actualmente el mismo informa proyecta un total de 298197 personas 
El Municipio de Armenia, construyó el Plan Municipal de Desarrollo 2008- 2012, en tal apuesta política e institucional se propuso dentro de sus objetivos: "Mejorar las condiciones de uso y apropiación, sostenibilidad y competitividad del territorio, mediante un adecuado proceso de planificación, el fortalecimiento de la gestión ambiental y la aplicación de instrumentos de gestión para el desarrollo territorial".

Existe una necesidad manifiesta de acudir a investigaciones que propendan por dar un salto hacia la consolidación de información referente a los resultados de un territorio, generada por evaluaciones desde una perspectiva integral para develar, de forma posterior a la ejecución de los programas y proyectos propios del instrumento de planeación, los cambios o avances en la condiciones de vida de la población.

En consonancia con lo anterior, el objetivo de la investigación consiste en evaluar el plan de desarrollo de la ciudad de Armenia, desde la dimensión ambiental, en el periodo 2008-2011.

La pregunta problemica que dio lugar a la investigación es ¿Cuál es el resultado del plan de desarrollo de la ciudad de Armenia en el periodo 2008-2011, desde la dimensión ambiental?

\section{DISEÑO METODOLÓGICO}

La investigación abordada se puede concebir como descriptiva, toda vez que acude a la implementación de indicadores en el Plan de Desarrollo 2008-2011, en aras de evaluar si se presentó un cambio en las condiciones de vida de la población de Armenia.

En primer lugar se describió las condiciones previas a la adopción del Plan de Desarrollo 2008-2011; relacionando los programas y subprogramas pertinentes a la investigación, efectuados en el marco del plan de desarrollo del periodo 2004-2007. En segundo lugar, se analizaron los programas y subprogramas que se implementaron con ocasión al Plan de Desarrollo 2008-2011 en el municipio de Armenia y que se ajustan a la dimensión abordada, estableciendo ciertos logros alcanzados en la sociedad, de forma tal que se reconozcan las escenarios alcanzados producto de la ejecución del instrumento de política pública.

Finalmente, se evaluó el escenario ex post al plan de desarrollo en 2008-2011, en la dimensión ambiental; la cual se llevó a cabo a partir de la aplicación de indicadores, en aras de establecer posibles cambios en las condiciones de vida de la población.

El diseño metodológico se fundamentó en la gestión integral de programas sociales orientadas a resultados (Unesco, 1999), mediante la aplicación de dicha técnica se puede conocer si las acciones implementadas a partir de la aplicación del instrumento de política ha ocasionado un cambio importante en las condiciones de vida (ambiental) en el tiempo analizado. De esta manera se pudo realizar una caracterización del plan de desarrollo y sus componentes, analizando información en los dos periodos mencionados, para inferir, al concluir la evaluación, si las variables estudiadas, entre las que se destacan, corredores urbanos con problemas de orden ecológico, consumo per cápita de agua, confluyeron en 
tres posibles escenarios a saber: resultados positivos, ninguna modificación o resultados negativos.

Los indicadores desarrollados permitieron obtener información clara que da cuenta de la realidad ambiental del Municipio, como una posibilidad para establecer un marco de referencia que permita a futuro, trazar acciones que coadyuven a consolidar escenarios de sostenibilidad. Para el desarrollo de la investigación se acudió fundamentalmente a la información suministrada por las diferentes dependencias y/o unidades administrativas de la Alcaldía Municipal y la Corporación Autónoma Regional del Quindío, entidad que regula el medio ambiente y los recursos naturales renovables.

Para tal fin, se ofició de manera formal a las unidades responsables de rendir la información, donde se obtuvo respuesta por escrito que posibilitó desarrollar la presente investigación.

\section{EVALUACIÓN DEL PLAN DE DESARROLLO EN EL MUNICIPIO DE ARMENIA EN LA DIMENSIÓN AM- BIENTAL DE LA SOSTENIBILIDAD, PERIODO 2008- 2011}

Una vez identificados aquellos subprogramas que tienen relación conexa o afín con el medio ambiente, se seleccionaron aquellos que se encuentran presentes en ambos planes de desarrollo bajo la misma denominación o que aun cuando reciben nombres diferentes tienen características comunes, a fin de establecer semejanzas entre ellos, bajo el entendido de que solo se podrán evaluar aquellos que tengan continuidad o que su espíritu sea similar en ambos instrumentos de planeación.

Dichos subprogramas, agrupados en categorías, dan cuenta de las dimensiones de la sostenibilidad; los cuales comprenden los indicadores desarrollados que se detallan a continuación:

\section{Dimensión ambiental}

Los fenómenos evaluados en el marco de la dimensión ambiental, los cuales están relacionados principalmente con la conservación del ecosistema, el estado de los recursos naturales, la gestión integral de residuos sólidos y del recurso hídrico, entre otros, los cuales tienen la intención de establecer el escenario ambiental del municipio y las condiciones que dan cuenta de la realidad observada.

La importancia de identificar el estado ambiental, está relacionada a la capacidad de la administración para armonizar todas aquellas actividades desarrolladas en el territorio, con respecto a la capacidad de recuperación y absorción del ecosistema, erigiendo una nueva racionalidad ambiental que reconozca los límites racionales de explotación para garantizar la regeneración de recursos y/o repoblación de especies, como parte fundamental del discurso de la sostenibilidad y el derecho al acceso a la base de recursos naturales para generaciones venideras. 
Entonces se establecieron las categorías que permitieran identificar la correcta gestión, uso y aprovechamiento de los recursos y las acciones adelantadas en cuanto a la gestión de riesgos de desastres.

\section{Categoría plan de gestión integral de residuos sólidos}

La categoría aludida hace referencia a la capacidad de la administración municipal para garantizar el aprovechamiento y valorización de los residuos sólidos (Indicadores: a) (residuos sólidos aprovechados/total residuos generados) * 100; b) (residuos sólidos reciclados/total residuos generados) * 100), evidenciando la adopción de procesos donde se privilegie la recirculación de materias primas como una posibilidad para hacer un uso eficiente de los recursos naturales.

Tabla 2.

\begin{tabular}{|c|c|c|}
\hline \multirow{5}{*}{ 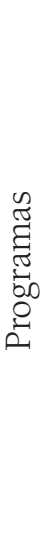 } & Plan de Desarrollo 2004-2007 & Plan de Desarrollo 2008-2011 \\
\hline & \multirow[t]{4}{*}{$\begin{array}{l}\text { Manejo Integral de Residuos } \\
\text { Sólidos. }\end{array}$} & $\begin{array}{l}\text { Recuperación, aprovechamiento y disposición } \\
\text { final de residuos sólidos, con una perspectiva } \\
\text { regional y soportado en el desarrollo institu- } \\
\text { cional y el fortalecimiento de las relaciones } \\
\text { empresa-usuario-operador. }\end{array}$ \\
\hline & & $\begin{array}{c}\text { Educación para la gestión integral de residuos } \\
\text { sólidos. }\end{array}$ \\
\hline & & $\begin{array}{l}\text { Investigación y desarrollo para la gestión inte- } \\
\text { gral de residuos sólidos. }\end{array}$ \\
\hline & & Residuos especiales. \\
\hline
\end{tabular}

Fuente: Elaboración propia

De acuerdo con el informe "Estado de los Recursos Naturales y del Ambiente" (Contraloría Municipal de Armenia, 2014), en lo que respecta al reciclaje aducen que: "Empresas Públicas de Armenia no cuenta con un registro pormenorizado de ventas de subproductos de RSM recuperados, ya que en el Municipio no existe un centro de acopio de manejo directo de EPA que le permita consolidar estadísticas al respecto. A pesar que son muchos los centros de acopio en Armenia, EPA ESP, no registra este tipo de variables en sus labores".

El informe en cuestión asevera igualmente que la disposición final de los residuos sólidos en el relleno sanitario, corresponde al total de residuos que son recolectados a los usuarios del servicio, así como las fracciones resultantes del barrido y aseo realizado a las vías públicas y parques.

Se solicitó del mismo modo a dicha entidad un informe al respecto, a lo cual se recibió un oficio donde manifiestan que durante el periodo de tiempo aludido el operador encargado de la Gestión de Aseo fue la empresa privada Servigenerales, por lo cual no cuentan con información al respecto. Es importante aclarar que Servigenerales ya no está ubicada en la ciudad de Armenia, por cuanto el servicio en mención fue recuperado recientemente por las Empresas Públicas de Armenia E. S. P. Tal situación permite establecer entonces que 
en la ciudad de Armenia no se llevan a cabo procesos de aprovechamiento y valorización de residuos sólidos.

\section{Categoría agua potable y saneamiento básico}

Dicha categoría se circunscribe a los proyectos adelantados con la intención de incrementar la cobertura en el acceso del sistema de saneamiento básico, el manejo integral del recurso hídrico y la optimización en su uso (Indicadores: (Población con acceso a métodos de acueducto y alcantarillado /Total población) * 100; b) (Fuentes receptoras monitoreadas/ total fuentes receptoras) * 100 (Monitoreo del 100\%); c) Consumo per cápita de agua (Total consumo de agua potable en $\mathrm{m}^{3}$ /Número de usuarios).

Tabla 3.

\begin{tabular}{|c|c|c|}
\hline & Plan de Desarrollo 2004-2007 & Plan de Desarrollo 2008-2011 \\
\hline & $\begin{array}{c}\text { Conservación de recursos hídricos en } \\
\text { el campo y en la zona urbana. }\end{array}$ & Manejo y uso eficiente del agua. \\
\hline & Manejo Integral del Recurso Hídrico. & $\begin{array}{l}\text { Recurso hídrico como eje } \\
\text { articulador de la planificación y la } \\
\text { integración territorial }\end{array}$ \\
\hline & Saneamiento Básico. & Ahorro y uso eficiente del agua. \\
\hline$\tilde{\tilde{Z}}$ & $\begin{array}{c}\text { Eficiencia en la prestación de } \\
\text { los servicios de acueducto y } \\
\text { alcantarillado. }\end{array}$ & Expansión de los servicios. \\
\hline .ี & $\begin{array}{l}\text { Solidaridad en Servicios Públicos } \\
\text { (Municipio-FSR). }\end{array}$ & Reposición de infraestructura. \\
\hline & $\begin{array}{c}\text { Construcción y Reposición de Redes } \\
\text { de Acueducto y Alcantarillado }\end{array}$ & $\begin{array}{c}\text { Rehabilitación de infraestructura y } \\
\text { contingencia, cultura y gestión del } \\
\text { riesgo. }\end{array}$ \\
\hline & & $\begin{array}{l}\text { Planeación técnica para el } \\
\text { desarrollo de los servicios. }\end{array}$ \\
\hline & & Subsidio a los servicios públicos. \\
\hline & & Expansión de la infraestructura. \\
\hline & & $\begin{array}{c}\text { Reposición y rehabilitación de in- } \\
\text { fraestructura. }\end{array}$ \\
\hline
\end{tabular}

Fuente: Elaboración propia

\section{Fuentes receptoras monitoreadas}

De acuerdo con la información suministrada por la Corporación Autónoma Regional del Quindío, durante la vigencia 2007 y 2011 se monitorearon el 100\% de las fuentes que abastecen de agua al acueducto Municipal de Armenia, para el caso particular se cuenta únicamente con una fuente: el río Quindío. Por lo tanto es prudente afirmar que no se perciben cambios o afectaciones a la sociedad de un periodo a otro. 
Gráfico 1. Usuarios (registrados y/o reportados) con acceso al sistema de acueducto y alcantarillado

\section{Usuarios con acceso a saneamiento basico}

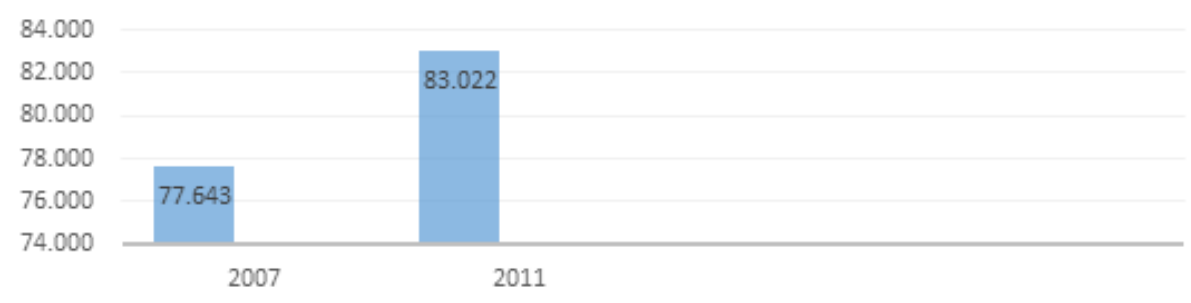

Fuente: Elaboración propia

En el 2007 las Empresas Públicas de Armenia (EPA, E. S. P.) reportaron un total de 77643 usuarios en condición de legalidad, para el año 2011 incrementó a 83022 usuarios, dicha situación obedece principalmente a un incremento significativo en los usuarios del sector comercial y residencial ((Fuentes receptoras monitoreadas/total fuentes receptoras) $* 100)$.

En lo que respecta al incremento de los usuarios residenciales se debe estimar que parte de ese incremento es motivado por el fenómeno inmobiliario que en los últimos años ha disparado las tasas de urbanización y edificación en Armenia, como bien reportan las cifras oficiales.

Sin embargo, tampoco se puede desconocer los esfuerzos realizados por la administración municipal en los últimos años y que han permitido masificar el servicio de saneamiento básico en algunas zonas antes desprovistas.

Gráfico 2. Consumo per cápita de agua: Total consumo de agua potable en $\mathrm{m}^{3}$ / Número usuarios

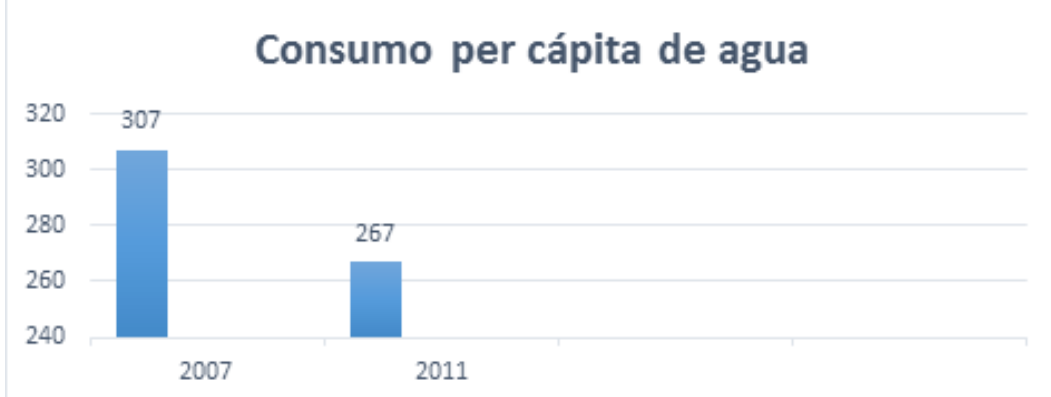

Fuente: Elaboración propia

Año 2007: $23850320 \mathrm{~m}^{3} / 77643$ usuarios

Año 2011: $22152356 \mathrm{~m}^{3} / 83022$ usuarios

El consumo per cápita anual de agua en Armenia para la vigencia 2007 es de $307 \mathrm{~m}^{3}$, para el 2011 la cifra se redujo a $267 \mathrm{~m}^{3}$. De acuerdo con la Superintendencia de Servicios 
Públicos Domiciliarios de Colombia, dichas cifras se ubican en un rango de tolerancia aceptable por su semejanza al de ciudades como Pereira, la cual tiene un consumo promedio anual por suscriptor de $264 \mathrm{~m}^{3}$, igualmente el consumo de la ciudad, se ubica por debajo de otras con mayor densidad demográfica como Cali y Bogotá. De acuerdo con la información suministrada por la Subgerencia de Aguas y Comercial se puede establecer que el consumo total de agua durante los últimos años ha presentado una tendencia variable, ubicándose en un rango entre 24 y 21 millones de metros cúbicos anuales en Armenia. En cuanto al número de usuarios, se observa un comportamiento al alza, ocasionado principalmente por el incremento en los usuarios comerciales y residenciales.

\section{Categoría manejo y conservación integral de los recursos naturales}

Los subprogramas desarrollados para ambos planes de desarrollo en el marco de la categoría en mención, se formularon con el objetivo de reconocer entre otros factores el cumplimiento de la normatividad ambiental, en particular el artículo 111 de la Ley 99 de 1993, del mismo modo que las acciones para la recuperación y conservación de ecosistemas estratégicos por sus servicios ambientales (a) (Superficie acumulada en hectáreas atendidas con acciones de reforestación y restauración de suelos/ Superficie acumulada en hectáreas deforestadas)*100; b) (Total de corredores urbanos con problemas de orden ecológico/ total corredores urbanos)*100; c) (No predios adquiridos en áreas estratégicas para la conservación del recurso hídrico según Ley 99 de 1993 / No predios planeados para adquirir) * 100; d) (No predios adquiridos en áreas estratégicas para la conservación del recurso hídrico según Ley 99 de 1993 / total predios ubicados, identificados y priorizados en áreas estratégicas para la conservación del recurso hídrico) * 100.

Tabla 4.

\begin{tabular}{|c|c|c|}
\hline \multirow{11}{*}{ 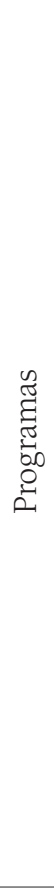 } & Plan de Desarrollo 2004-2007 & Plan de Desarrollo 2008-2011 \\
\hline & $\begin{array}{l}\text { Sistema de áreas de interés (Artículo } \\
111 \text { Ley 99/93) }\end{array}$ & $\begin{array}{l}\text { Recuperación y conservación de fuentes abaste- } \\
\text { cedoras, microcuencas urbanas y rurales. }\end{array}$ \\
\hline & Ecosistemas Estratégicos Municipales & $\begin{array}{l}\text { Infraestructura ambiental para las microcuencas } \\
\text { y corredores ambientales urbanos. }\end{array}$ \\
\hline & $\begin{array}{c}\text { Recuperación y Mantenimiento de } \\
\text { Drenajes. }\end{array}$ & $\begin{array}{c}\text { Conservación de la biodiversidad, el paisaje cafe- } \\
\text { tero y la recuperación de la calidad del aire para } \\
\text { un ambiente sano. }\end{array}$ \\
\hline & Proyectos Ambientales. & $\begin{array}{l}\text { Investigación y conocimiento para el uso sosteni- } \\
\text { ble de la biodiversidad. }\end{array}$ \\
\hline & Parques y zonas verdes de la ciudad & Instrumentos de planificación territorial. \\
\hline & Manejo y conservación de áreas prote- & Instrumentos de gestión y financiación. \\
\hline & gidas y zonas de alto riesgo & $\begin{array}{l}\text { Instrumentos de seguimiento y evaluación del } \\
\text { desarrollo territorial urbano y rural. }\end{array}$ \\
\hline & & Gestión, control y seguimiento urbano. \\
\hline & & Gestión de proyectos urbanos especiales. \\
\hline & & Manejo integral de drenajes urbanos. \\
\hline
\end{tabular}

Fuente: Elaboración propia 


\section{- Superficie acumulada en hectáreas atendidas con acciones de reforestación y restauración}

De conformidad con la información suministrada por la autoridad ambiental departamental (CRQ), se pudo acceder al dato de las hectáreas reforestadas para las vigencias objeto de análisis, correspondiendo a 5 hectáreas para el año 2007 e igual cantidad para el año 2011. Respecto de las áreas deforestadas según informe de la misma entidad al cierre del año 2014(fecha de culminación de la investigación), no se ha consolidado esta información, en razón a la no realización de análisis multitemporal de imágenes que permitan calcular las áreas de bosque disminuidas de forma anual.

Como alternativa de acceso a esta información se realizó consulta a través de las estadísticas del Ministerio de Ambiente y Desarrollo Sostenible, por lo cual fue posible establecer que esta entidad no cuenta con estudios sobre tasas anuales de deforestación para el periodo de análisis, toda vez que el primer informe nacional se elaboró para la vigencia 2013, siendo publicado el 17 de octubre de 2014, por lo cual se hace improcedente el cálculo del indicador para las vigencia objeto de análisis de esta investigación.

\section{- Total de corredores urbanos con problemas de orden ecológico}

De acuerdo con información suministrada por el Departamento de Planeación Municipal, existen tres (3) corredores urbanos en la ciudad, los cuales en su totalidad se han visto afectados desde hace varias décadas por aguas residuales domesticas que causan contaminación.

Tal situación obedece a que los colectores no se encuentran enlazados en su totalidad a las Plantas de Tratamiento de Aguas Residuales, generando vertimientos directos de aguas domésticas en los corredores urbanos, alterando las condiciones del ecosistema en detrimento de los ciudadanos que habitan en áreas colindantes.

Dicha dependencia igualmente manifiesta que la administración municipal viene adelantando acciones para mitigar el impacto en aquellas microcuencas más contaminadas de la ciudad y que se encuentran en proceso de construcción al menos tres Plantas de Tratamiento de Aguas Residuales que conecten los diversos colectores en aras de subsanar y prevenir a futuro la contaminación objeto de discusión.

\section{- Predios adquiridos en áreas estratégicas para la conservación del recurso hídrico}

De acuerdo a la información suministrada por el Departamento Administrativo de Planeación Municipal y el Informe "Estado de los Recursos Naturales y del Ambiente (Contraloría Municipal de Armenia, 2014), durante el periodo de tiempo analizado (vigencia 2011) no se realizó adquisición de predios en zonas estratégicas para la conservación del recurso hídrico por parte de la administración municipal.

Es importante manifestar que hasta la fecha de publicación de la presente investigación, la administración municipal ha adquirido seis predios adyacentes a la cuenca del 
río Quindío, los cuales se encuentran en áreas de vital importancia para la protección de recursos hídricos que surten el acueducto del municipio de Armenia.

El Municipio de Armenia realizó la adquisición de los predios adquiridos (La Zulia, Morro Azul, La Estrella, La Cabaña 1, La Cabaña 2, La Esperanza) con ocasión al artículo 111 de la Ley 99 de 1993, el cual establece que los municipios deberán destinar un porcentaje igual o superior al $1 \%$ de sus ingresos corrientes para la compra de predios ubicados en áreas estratégicas para la conservación de la oferta hídrica que abastece al acueducto de Armenia.

De acuerdo con la información suministrada por las fuentes citadas anteriormente, dichas inversiones alcanzaron una inversión total de US\$1988103421, de los cuales US\$ 984103421 fueron destinados en la vigencia 2013.

\section{Categoría gestión local del riesgo}

La gestión local del riesgo (Indicador: (Número total población afectada por desastres en el Municipio de Armenia)) ha de ser una de las labores de mayor importancia en el marco de la sostenibilidad, toda vez que mediante la adopción de estrategias preventivas, se evitan posibles desastres que atenten contra la seguridad de los habitantes, garantizando entonces el derecho fundamental a la vida.

Los indicadores desarrollados en el marco de la gestión del riesgo pretenden reconocer el número de afectados, con ocasión a los diferentes desastres naturales u otras emergencias que hayan provocado alteraciones en las condiciones de vida de la población, así como las diferentes acciones adelantadas en aras de mitigar los impactos causados en las posibles víctimas.

\section{Tabla 5.}

\begin{tabular}{|c|c|c|}
\hline \multirow{8}{*}{ 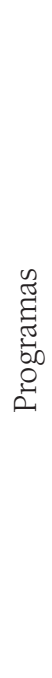 } & $\begin{array}{l}\text { Plan de Desarrollo } \\
\text { 2004-2007 }\end{array}$ & Plan de Desarrollo 2008-2011 \\
\hline & \multirow{7}{*}{$\begin{array}{c}\text { Prevención y atención } \\
\text { de } \\
\text { desastres. }\end{array}$} & $\begin{array}{l}\text { Adelantar procesos para la disminución de vulnerabilidades y miti- } \\
\text { gación de riesgos existentes }\end{array}$ \\
\hline & & $\begin{array}{l}\text { Ejecutar procesos para la prevención de riesgos potenciales y cultura } \\
\text { para la gestión del riesgo }\end{array}$ \\
\hline & & Fortalecimiento institucional para la gestión integral del riesgo. \\
\hline & & $\begin{array}{l}\text { Gestión para la identificación y priorización de los riesgos de emer- } \\
\text { gencias desastres }\end{array}$ \\
\hline & & $\begin{array}{l}\text { Articulación intersectorial para el desarrollo de planes preventivos, } \\
\text { de mitigación y superación de las emergencias y desastres }\end{array}$ \\
\hline & & $\begin{array}{l}\text { Fortalecimiento institucional para la respuesta territorial ante las si- } \\
\text { tuaciones de emergencias y desastres }\end{array}$ \\
\hline & & Acciones de fortalecimiento de la red de urgencias \\
\hline
\end{tabular}

Fuente: Elaboración propia 
Gráfico 3. Total de población afectada por desastres en el Municipio de Armenia

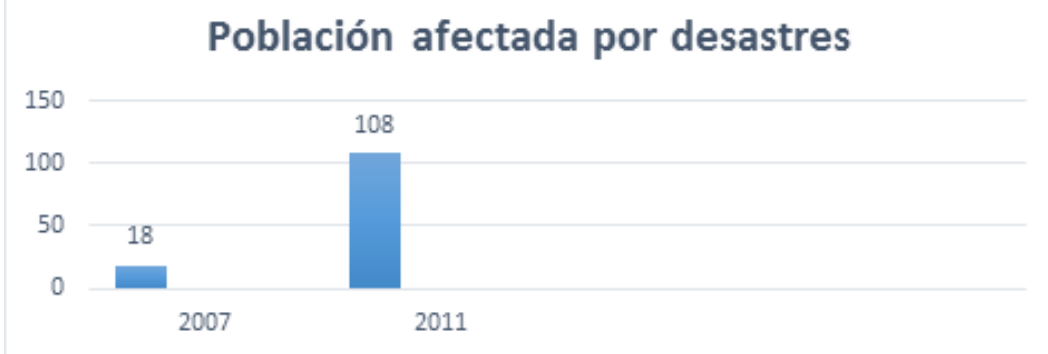

Fuente: Elaboración propia

Durante el año 2007 se vieron afectados 18 personas en emergencias reportadas al Cuerpo Oficial de Bomberos, durante el 2011 la cifra aumento a 108.

Es de aclarar que los afectados durante la vigencia 2007 se deben fundamentalmente a colapsos de estructuras y deslizamientos, mientras que para el año 2011 se debió en orden de importancia a incendios, vendavales y deslizamientos, sin que se presentaran víctimas fatales en ambas vigencias.

Según información suministrada por las autoridades el incremento con ocasión a las fuertes temporadas de lluvias y vendavales que causaron movimientos en masa, dio lugar a la reubicación de 17 familias provenientes en su mayoría en asentamientos subnormales.

Es de aclarar que de acuerdo con la Oficina de Gestión de Riesgo de Desastres de la Alcaldía de Armenia en ambos casos se atendieron al 100\% de los afectados, suministrando ayuda humanitaria y que en el caso específico de las personas reubicadas se les otorgó el auxilio de arrendamiento durante el 2011 para luego ser reubicados en viviendas propias en la vigencia 2012.

La administración municipal está en mora de aplicar con mayor rigurosidad medidas que conlleven a reubicar a personas que habitan en zonas de alto riesgo, dichas actuaciones deberán estar enmarcadas en los lineamientos establecidos por el Plan de Ordenamiento Territorial,

Se espera entonces que las acciones adelantadas en cuanto a prevención y mitigación de riesgos y que están en cabeza de la Oficina de Gestión de Riesgo de Desastres, contribuyan significativamente a reducir el número de afectados por siniestros y otros factores de riesgo.

Con ocasión a la dimensión ambiental, se puede establecer a partir de la investigación, un escenario que devela fallas en la adopción de medidas eficaces para dar solución a problemáticas como las referidas a la inexistencia de acciones para el aprovechamiento y valorización de residuos sólidos, la no adquisición de predios estratégicos para la conservación de la oferta hídrica, el estado de contaminación de los corredores biológicos producto de un déficit en la articulación de los colectores con sistemas de tratamiento de aguas residuales y el incremento en la población afectada por desastres, fenómeno el cual 
incrementó el número de desplazamientos intra municipales por movimientos en masa, vendavales, entre otros.

Lo anterior pone en entredicho la efectividad de los programas y proyectos puestos en marcha en el marco del plan de desarrollo evaluado, siendo prioritario que los resultados esbozados en la investigación sirvan como insumo en la toma de decisiones del ente municipal para dar una solución pronta y eficaz a los factores críticos descritos anteriormente

\section{DISCUSIÓN DE RESULTADOS}

El plan de desarrollo para el periodo 2008-2011, como instrumento de planeación fue concebido bajo los pilares de la sostenibilidad; como una posibilidad para alcanzar un desarrollo incluyente y justo en los armenios. Para alcanzar tan noble fin se desarrollaron, entre otros, programas y subprogramas concebidos hacia lo ambiental, los cuales a partir de los resultados develados por la investigación, serán objeto de análisis y discusión sobre los resultados en la sociedad.

En la dimensión ambiental, de diez indicadores propuestos se calcularon nueve, de los cuales en seis indicadores no se presentaron cambios de un periodo a otro.

Los indicadores en los que no se presentaron cambios son:

- (Residuos sólidos aprovechados/total residuos generados)

- (Residuos sólidos reciclados/total residuos generados)

- (No predios adquiridos en áreas estratégicas para la conservación del recurso hídrico según Ley 99 de 1993 / No predios planeados para adquirir)

- (No predios adquiridos en áreas estratégicas para la conservación del recurso hídrico según Ley 99 de 1993 / total predios ubicados, identificados y priorizados en áreas estratégicas para la conservación del recurso hídrico)

- (Total de corredores urbanos con problemas de orden ecológico/ total corredores urbanos)

- (Fuentes receptoras monitoreadas/ total fuentes receptoras)

Sin embargo, se debe aclarar que para los primeros cinco indicadores el estado de equilibrio paradójicamente representa un resultado negativo, dado sus implicaciones en las condiciones de la sostenibilidad, los cuales se explican a continuación:

En lo que respecta a los residuos sólidos, la problemática se suscita debido a la falta de acciones encaminadas a garantizar un aprovechamiento y/o valorización de las fracciones de materiales, por lo tanto, la gestión de residuos se circunscribe fundamentalmente al destino final en relleno sanitario y en otros casos como el de residuos hospitalarios a incineración.

Por su parte, la no adquisición de predios estratégicos para la conservación de la oferta hídrica constituye un incumplimiento de la Ley 99 de 1993 y sus demás leyes o decretos que lo modifican o reglamentan, toda vez que producto del estado actual de los recursos 
no renovables se hace indispensable el destinar parte de los ingresos del ente municipal para la conservación y recuperación de ecosistemas estratégicos.

Asimismo, en el caso de los corredores urbanos, se pudo establecer un estado de contaminación y que es recurrente desde hace varias décadas por cuenta de la falta de articulación de los colectores con sistemas de tratamiento de aguas residuales, lo que origina un vertimiento directo de aguas servidas que alteran las condiciones del ecosistema y los servicios ambientales provistos.

Se observa además resultados negativos en otro indicador, a causa del incremento en la población afectada por desastres, fenómeno el cual incrementó el número de desplazamientos intramunicipales por movimientos en masa, vendavales, entre otros, que deja entrever las falencias del sistema de gestión del riesgo municipal, en cabeza de la Secretaría de Gobierno, toda vez que sus acciones no fueron eficaces para lograr disminuir los focos de riesgo existentes y evitar posibles desastres en la población, principalmente de aquellos que habitan en asentamientos subnormales.

Por otro lado, se alcanzó un logro positivo en dos indicadores, debido al incremento en el número de usuarios que acceden a saneamiento básico en la ciudad y la reducción en el consumo per cápita de agua.

El primer fenómeno se puede explicar como una consecuencia de la actividad inmobiliaria que en los últimos años ha tenido un comportamiento favorable, la cual trae consigo mayor expansión urbanística y por ende un incremento en las tasas de cobertura de saneamiento básico.

En lo que respecta al promedio de consumo de agua, se puede concluir que a partir de las estrategias desarrolladas por el ente municipal se logró sensibilizar a la población en el consumo racional del recurso hídrico, logrando reducir ostensiblemente el promedio per cápita, como una posibilidad para conservar a futuro un recurso vital para la vida y garantizar su acceso a futuras generaciones. Se estima conveniente avanzar en campañas dirigidas hacia la población así como a los sectores económicos en general, en miras de alcanzar un consumo eficiente que redunde en menores tasas de aprovechamiento, dado que el incremento en la población y las incorrectas practicas industriales son factores que presionan constantemente al alza en detrimento de la sostenibilidad de la oferta hídrica municipal.

De acuerdo con lo esbozado anteriormente, se puede concluir que en la dimensión ambiental de nueve indicadores calculados, seis fueron negativos, dos positivos y finalmente uno no presento cambios de un periodo a otro, de tal suerte que al realizar una evaluación integral se hace evidente un resultado negativo para la dimensión ambiental, lo que sugiere a la administración municipal la toma de medidas para dar una solución pronta y eficaz a los problemas argumentados en la investigación, y que a su vez desestima las acciones adelantadas en el marco del plan de desarrollo evaluado por su poca trascendencia. 
Finalmente, resulta preocupante argumentar que a la luz de los resultados alcanzados, las acciones adelantas con ocasión al plan de desarrollo no fueron pertinentes y coherentes para alcanzar un escenario sostenible desde lo ambiental.

\section{CONCLUSIONES}

Los indicadores desarrollados posibilitaron evaluar los programas y subprogramas del plan de desarrollo, identificando avances o retrocesos en las condiciones de vida de la población en torno a la dimensión ambiental de la sostenibilidad.

El plan de desarrollo de la ciudad de Armenia, correspondiente al periodo 2008-2011, tomó como punto de referencia un diagnóstico que reveló el estado actual del territorio, donde se identificaron diversas problemáticas de índole, las cuales constituyeron el punto de partida de la agenda pública, en la que se consideró el fortalecimiento del capital social, generando condiciones de equidad social, sostenibilidad como objetivos rectores del plan para dar solución a las necesidades sentidas por la población.

En ese orden de ideas, la administración municipal formuló dentro del plan de desarrollo una serie de políticas para alcanzar los objetivos propuestos, entre las que se destacan las siguientes:

- Política de desarrollo ambiental sostenible

- Política de servicios públicos

- Política de seguridad alimentaria y nutricional

- Política de competitividad y productividad

- Política de emprenderismo, generación de empresas y empleo productivo

De acuerdo con los preceptos de la investigación, el plan de desarrollo 2008-2011 se concibió bajo una perspectiva de sostenibilidad, toda vez que propendió mediante los programas evaluados a una dinamización socioeconómica para fortalecer el desarrollo del territorio y, por supuesto, de los ciudadanos; pensado desde valores como la conservación del ecosistema, la justicia social, la resignificación del territorio y del ser humano como eje dinamizador de la sociedad.

Sin embargo, el desarrollo de la investigación permitió fijar una postura distinta en torno a la eficacia de los programas desarrollados, estableciendo entonces que los mismos dieron lugar a cambios negativos en las condiciones de vida de la población.

Sin embargo, tales resultados no ofrecen argumentos de peso para establecer el impacto en la calidad de vida de los armenios, para lo cual se hace necesario acudir al análisis de los resultados para los indicadores desarrollados en los programas de orden económico y ambiental, los cuales se presentan a continuación.

Para la dimensión ambiental de nueve indicadores calculados, dos presentaron avances favorables (usuarios con acceso al sistema de acueducto, alcantarillado y consumo de agua per cápita); uno sin generar alternaciones en la sociedad (fuentes receptoras moni- 
toreadas), mientras que en seis se observó un retroceso o impacto negativo (población afectada por desastres en el Municipio de Armenia, corredores urbanos con problemas de orden ecológico, dos relacionados con adquisición de predios para la conservación de fuentes hídricas que abastecen al municipio y otros dos que tienen que ver al aprovechamiento y valorización de residuos sólidos).

En lo que respecta a la población afectada por desastres, en el Municipio de Armenia, se presenta un comportamiento negativo debido al incremento de fenómenos de diverso orden como incendios, deslizamientos, entre otros, que causan alteraciones en el orden público y atentan contra la integridad de la población civil. Es responsabilidad del ente municipal aplicar medidas eficaces para revertir la tendencia al alza que se viene presentando últimamente.

Dicho fenómeno evidentemente constituye un incumplimiento de la política de desarrollo ambiental sostenible, particularmente, en el programa de gestión integral del riesgo y ocupación sostenible en el territorio, del mismo modo que los tres subprogramas que lo conforman, por cuanto dichos subprogramas estaban orientados hacia la minimización de las vulnerabilidades y la mitigación de los riesgos existentes, razón por la cual era de esperarse que se disminuyeran el número de afectados por desastres de diverso orden, caso contrario a las cifras presentadas en esta investigación.

Es importante argumentar que para el caso particular de corredores urbanos con problemas de orden ecológico, resulta preocupante reconocer la poca efectividad del programa de gestión de bienes y servicios ambientales, el cual hace parte de la política desarrollo ambiental sostenible; así como al programa de plan de saneamiento y manejo de vertimientos de la política de servicios públicos, toda vez que no se ha dado solución a una problemática ambiental que se viene suscitando hace varias décadas, siendo el crecimiento de la contaminación proporcional a la expansión urbanística de Armenia; lo anterior por cuanto existen tres corredores urbanos en la ciudad, los cuales en su totalidad son afectados por vertimientos directos de aguas residuales domesticas que alteran las funciones ecológicas del ecosistema. Se espera entonces que la administración municipal tome acciones expeditas que conlleven a la construcción e implementación de, por lo menos, tres plantas de tratamiento de aguas residuales que conecten la totalidad de los colectores, garantizando una correcta disposición de los residuos con miras a eliminar el foco de contaminación.

Por otro lado, de acuerdo con el programa de gestión de bienes y servicios ambientales, protección de la biodiversidad y al programa manejo integral del recurso hídrico que pertenecen a la política de desarrollo ambiental sostenible; a la Ley 99 de 1993 y demás que la modifiquen o reglamenten, se puede establecer que la administración municipal no cumplió con las metas fijadas, por lo cual está en mora de destinar mayores recursos para la adquisición de predios para la conservación de fuentes hídricas que abastecen al municipio como una posibilidad para su conversación, goce y disfrute de las generaciones venideras. 
Finalmente, es prioritario retomar los objetivos propugnados en el subprograma de recuperación, aprovechamiento y disposición final de residuos sólidos, que hacen parte del programa Plan de Gestión Integral de Residuos Sólidos, perteneciente a la política de servicios públicos, por cuanto hubo carencia de proyectos de desarrollo para el aprovechamiento de las fracciones de residuos que garantizarán una recirculación de materiales en aras de reducir la carga contaminante producto de la disposición en rellenos sanitarios.

Los seis indicadores de la dimensión ambiental con resultados adversos, del mismo modo que el incumplimiento de tres de los cuatro programas evaluados que conforman la política de desarrollo ambiental sostenible y también de dos de los tres programas de la política de servicios públicos que fueron seleccionados por la investigación, constituye a todas luces un resultado negativo para la dimensión en comento y que, lamentablemente, repercute sobremanera en el bienestar de la población.

De acuerdo con las categorías desarrolladas es evidente que los problemas no obedecen a fallas en la consideración de las políticas y programas del plan de desarrollo, habida cuenta que estas fueron orientadas hacia un escenario sostenible, siendo entonces un fiel reflejo de las falencias en la operativización de este, toda vez que el hecho de que no se haya revertido la tendencia negativa en la dimensión ambiental, en aspecto atinentes a la población afectada por desastres, corredores urbanos con problemas de orden ecológico, escasa o nula adquisición de predios para la conservación de fuentes hídricas que abastecen al municipio y el no aprovechamiento y valorización de residuos sólidos, ofrece razones para argumentar que los esfuerzos dirigidos por la administración municipal no apuntaron hacia el cumplimiento de las políticas y objetivos definidos en el instrumento de planeación.

Se espera finalmente que los resultados de esta investigación sirvan como una base para futuras investigaciones, que se deberán desarrollar preferiblemente para diferentes periodos de tiempo y replicadas en otros espacios territoriales; lo anterior permitirá que se pueda mapear la realidad de diferentes municipios y/o ciudades, como un insumo que sirva de referente en las discusiones de los planes de desarrollo, garantizando así programas y estrategias que coadyuven eficazmente a solucionar las falencias que hayan sido detectadas.

\section{REFERENCIAS BIBLIOGRÁFICAS}

Contraloría Municipal de Armenia. (2014). Estado de los Recursos Naturales y de Ambiente. pp. 227. Armenia.

Departamento Administrativo Nacional de Estadística. (2010). Información estadística: Proyecciones de población municipales por área 2005-2020. Bogotá: Dane.

Departamento Nacional de Planeación. (2011). Evaluación del Desempeño Integral de los Municipios. Bogotá: Imprenta Nacional de Colombia. 
Leff, E. (2011). Globalización, racionalidad ambiental y desarrollo sustentable. México: Siglo XXI Editores.

Lovelock, J. (1979). Gaia, una nueva visión de la vida sobre la tierra. Londres: Oxford University Press.

Naredo, J. (2009). Raíces económicas del deterioro ecológico y social. Más allá de los dogmas. España: Siglo XXI.

Neef, M. (1994). El desarrollo a escala humana. Barcelona: Icaria.

Redclift, M. (1996). Los conflictos del desarrollo y la crisis ambiental. Estados Unidos de América: Fondo de Cultura Económica.

Sen, A. (2000). Desarrollo y libertad. Buenos Aires: Editorial Planeta.

Unesco. (1999). Gestión integral de programas sociales orientada a resultados. Buenos Aires: FCE. 